\title{
INVESTIGAÇÃO EXPERIMENTAL DA CINÉTICA DE REMOÇÃO DE DEPÓSITOS DE PROTEINA DE LEITE USANDO REATOR TIPO TANQUE AGITADO
}

\author{
J. S. VIEIRA ${ }^{1}$, G. A. P. SILVA ${ }^{1}$, B. H. O. PINHEIRO ${ }^{1}$, C. A. ALVARES ${ }^{1}$ e R. GEDRAITE ${ }^{1}$ \\ ${ }^{1}$ Universidade Federal de Uberlândia, Faculdade de Engenharia Química \\ E-mail para contato: jessica_silva_viera@hotmail.com
}

\begin{abstract}
RESUMO - Este trabalho teve como objetivo avaliar a cinética de remoção de depósitos à base de proteínas de leite, considerando o emprego de reator tipo tanque agitado. $\mathrm{O}$ estudo foi baseado na técnica de limpeza tradicionalmente empregada em sistemas de limpeza CIP da indústria, envolvendo o emprego de solução detergente alcalina. $\mathrm{O}$ modelo identificado ajustado representou de maneira coerente o comportamento do sistema estudado. $\mathrm{O}$ fato dos valores da absorbância não terem sido coletados de maneira automática não interferiu de maneira significativa no ajuste do modelo matemático, haja vista o erro quadrático integrado calculado entre o resultado fornecido pelo modelo e aqueles obtidos no experimento ter sido inferior a 0,02227. Quando comparado com o processo de remoção envolvendo o escoamento da solução detergente em contato direto com a superfície incrustada o método usado neste trabalho se mostrou mais lento.
\end{abstract}

\section{INTRODUÇÃO}

A técnica de limpeza Clean-in-Place (CIP) é amplamente utilizada na indústria de alimentos e na indústria farmacêutica, dentre outras, visando evitar a parada de produção e a desmontagem parcial ou total dos equipamentos de processamento. Sabe-se que diversas pesquisas têm sido realizadas nos últimos anos para procurar formas de melhorar $o$ procedimento (Bansal e Chen, 2006; Bremer et al., 2006). Tanto a tensão de cisalhamento aplicada quanto as reações químicas entre o fluido em escoamento e os depósitos formados são importantes na remoção de incrustações. A indústria de laticínios sofre com problemas específicos associados com a limpeza de equipamentos de processo, incluindo trocadores de calor (Georgiadis et al., 1998). Atualmente, o procedimento de limpeza envolve a lavagem do equipamento com solução detergente alcalina a fim de remover adequadamente os resíduos e incrustações que tendem a favorecer a contaminação. Depois de terminada a limpeza, o equipamento de processo deve ser enxaguado criteriosamente para remover todos os vestígios dos agentes de limpeza.

Este trabalho apresenta o resultado experimental da cinética de remoção de resíduos de leite em seção de testes, conduzida em reator de mistura com agitação, e respectivo modelo matemático, representativo da etapa de limpeza alcalina tipicamente utilizada em sistemas de limpeza CIP na indústria alimentícia. 


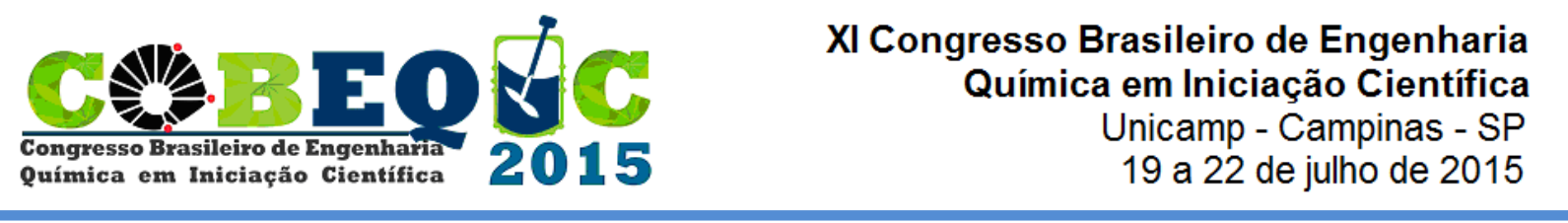

\section{MATERIAIS E MÉTODOS}

Os experimentos foram conduzidos usando o sistema apresentado na Figura 1, consistindo de um béquer de $1 \mathrm{~L}$ e respectivo sistema de agitação com rotação ajustável. Para este trabalho foi utilizada a rotação de $400 \mathrm{rpm}$. Esse motor é o responsável por aplicar um sinal no sistema, nesse caso, um degrau unitário, quando acionado a partir do repouso. A partir das respostas obtidas com esse sinal, foram identificados modelos aproximados, de baixa ordem, que descrevem o comportamento dinâmico do processo de remoção dos resíduos.

A superfície usada para os testes de remoção de resíduos foi uma placa em aço inoxidável, de dimensões aproximadas de $8 \times 8 \mathrm{~cm}$. O processo de incrustação foi realizado a partir do aquecimento da placa submersa em leite de vaca comprado em supermercado. Pegou-se a placa de inox inicialmente limpa e mergulhou-se a mesma em $200 \mathrm{~mL}$ de leite puro em um Becker de 2 litros. Levou-se este mesmo Becker já com a placa e o leite em banho-maria a uma temperatura de $60^{\circ} \mathrm{C}$ por 40 minutos. Ao fim do aquecimento, observou-se visualmente que uma camada de leite incrustado havia sido formada nas faces da placa. Feito isto, retirou-se a placa cuidadosamente do béquer e levou-se a mesma à estufa, por aproximadamente 24 horas.

O passo seguinte consistiu na limpeza da placa incrustada, a partir do processo CIP proposto neste trabalho, denominado CIP estático. A solução detergente alcalina utilizada foi obtida pela dissolução de hidróxido de sódio em água, na concentração de $0,5 \%$ em massa. Foi preparado $1 \mathrm{~L}$ da mesma no dia em que os testes foram realizados. A placa foi submersa no interior do béquer, na temperatura ambiente, e ajustada à rotação constante de $400 \mathrm{rpm}$ no agitador. Iniciado o processo de remoção dos resíduos de leite, foram retiradas amostras de aproximadamente 3 a $4 \mathrm{~mL}$ da solução, de minuto em minuto, para que as mesmas pudessem ser analisadas no espectrofotômetro UV, utilizando o comprimento de onda de $500 \mathrm{~nm}$.

Figura 1 - Protótipo de sistema CIP estudado

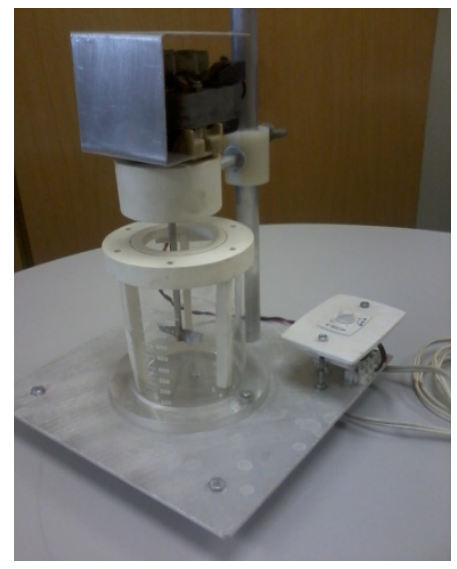


Realizaram-se dois experimentos seguindo o procedimento experimental citado. Os dados foram coletados manualmente, e a partir dos mesmos, curvas empíricas de concentração versus tempo foram obtidas.

Os modelos semi-empíricos identificados (Gormezano, 2007; Reis et al, 2012) foram utilizados como ponto de partida para a análise dos dados experimentais obtidos. Neste trabalho os modelos identificados levaram também em consideração o comportamento de variação contínua e gradual de aumento da concentração de resíduo solubilizado ao longo do tempo, para o processo conduzido com o escoamento da solução detergente através dos tubos de trocador de calor de feixe tubular.

O sistema foi modelado com base em função de transferência do tipo primeira ordem com tempo morto (FOPDT, em inglês). Eles foram simulados no ambiente Simulink ${ }^{\circledR}$ do aplicativo Matlab ${ }^{\circledR}$ e os resultados obtidos comparados com os dados experimentais coletados. A Figura 2 apresenta um diagrama de simulação típico utilizado nas análises desenvolvidas neste trabalho.

Figura 2 - Diagrama de simulação do processo estudado

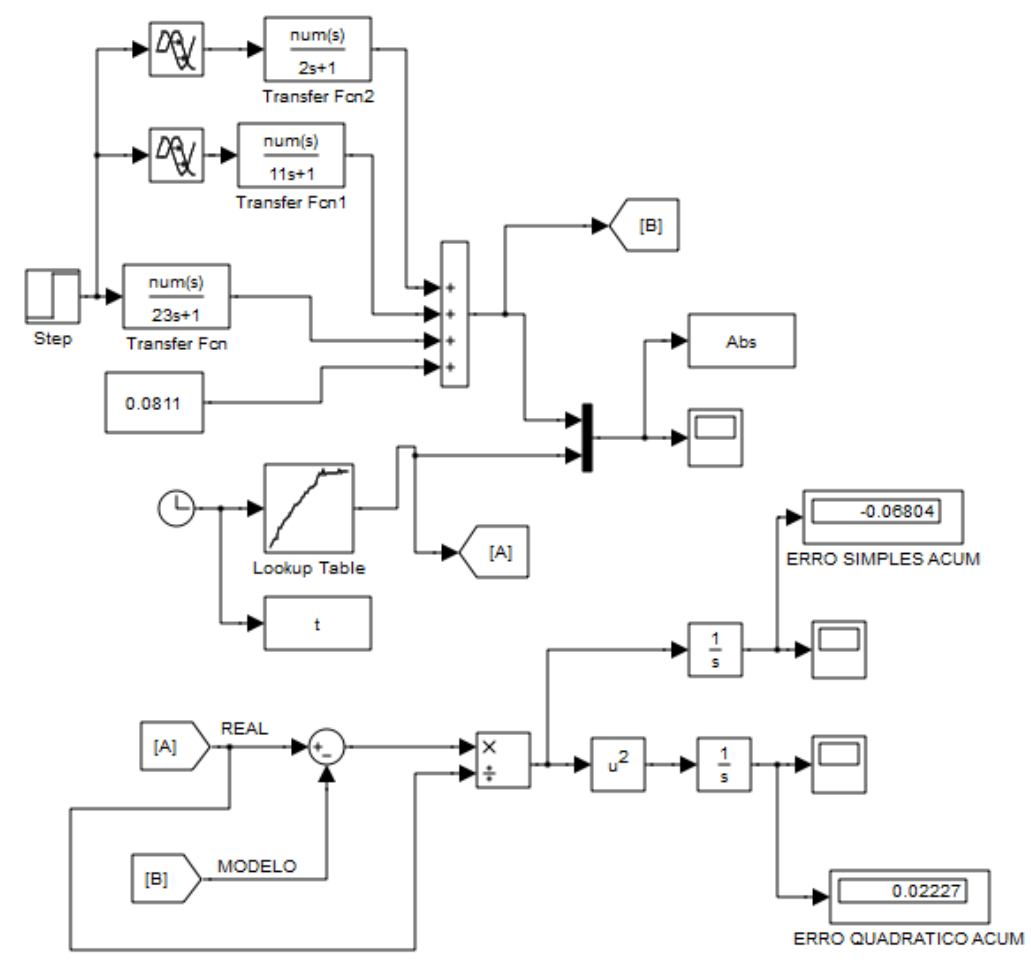

\section{RESULTADOS E DISCUSSÃO}

Na Figura 3 são apresentados os comportamentos experimental e simulado no aplicativo Matlab/Simulink ${ }^{\circledR}$ para o experimento realizado. Os parâmetros dos modelos identificados do 
tipo FOPDT são apresentados na Tabela 1, e foram obtidos com base no método proposto por Sundaresan e Krishnaswamy (1978).

Tabela 1 - Parâmetros dos modelos FOPDT identificados

\begin{tabular}{ccc}
$\begin{array}{c}\text { Ganho } \\
\text { (absorbância/rpm) }\end{array}$ & $\begin{array}{c}\text { Atraso de Transferência } \\
(\min .)\end{array}$ & $\begin{array}{c}\text { Atraso de Transporte } \\
(\mathrm{min} .)\end{array}$ \\
\hline 3,3867825E-04 & 23 & 0 \\
\hline 4,648525E-05 & 11 & 13 \\
\hline $1,6601875 \mathrm{E}-06$ & 2 & 30 \\
\hline
\end{tabular}

Figura 3 - Comportamentos experimental e simulado para o experimento

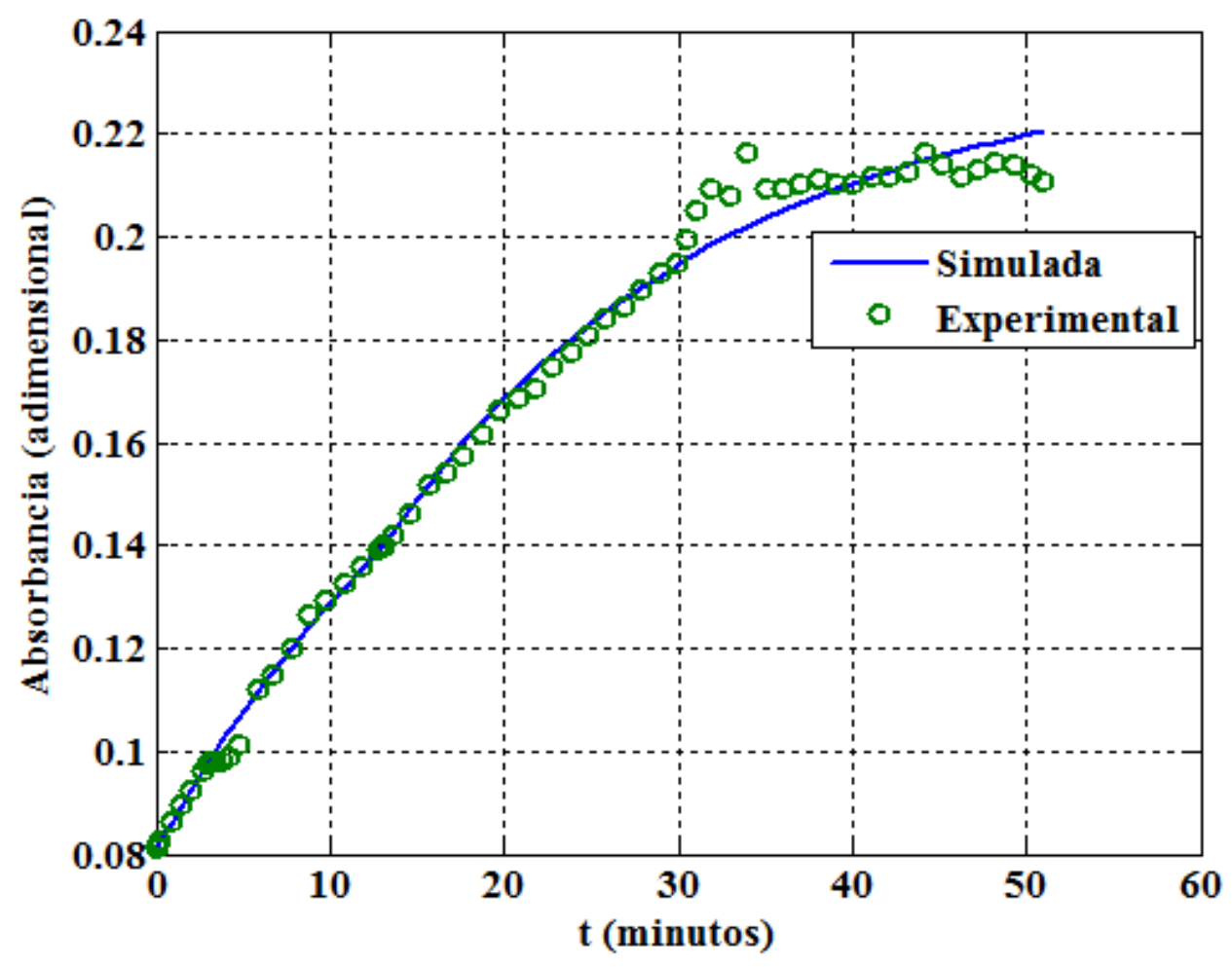

O critério utilizado para avaliar o grau de ajuste dos modelos empíricos aos dados experimentais correspondentes foi o ISE - Integrated Square Error (Erro Quadrático Integrado), tipicamente empregado para avaliar o desempenho de malhas de controle industriais, o qual apresenta a característica de penalizar mais fortemente os grandes erros (Garcia, 2005; Ogata, 2000). 
O valor correspondente ao erro quadrático integrado entre os valores experimentais e os respectivos valores simulados, obtido de maneira iterativa, usado como critério para o ajuste do modelo identificado neste trabalho foi igual a 0,02227 ou $2,23 \%$.

\section{CONCLUSÕES}

Considerando os resultados obtidos no experimento, pode-se concluir que o emprego da remoção dos resíduos em reator do tipo tanque agitado apresentou atraso de transferência significativamente maior do que aquele verificado em sistemas com escoamento da solução através da superfície a ser limpa.

O atraso de transferência para o sistema estudado neste trabalho foi aproximadamente igual a 780 segundos, ao passo que em sistemas envolvendo escoamento da solução de detergente alcalino, este valor é reduzido para aproximadamente 60 segundos. Esta constatação confirma a importância da turbulência provocada pelo fluido em escoamento na remoção da camada de resíduos depositada sobre a superfície.

\section{AGRADECIMENTOS}

Os autores agradecem à UFU e à FAPEMIG (Fundação de Amparo à Pesquisa do Estado de Minas Gerais) pelos recursos concedidos e apoio em pesquisas nos projeto $\mathrm{N}^{\mathrm{o}}$ TECAPQ-02100-12 (Estudo da Otimização de Sistema de Limpeza CIP).

\section{REFERÊNCIAS}

BANSAL, B., CHEN, X. D. 2006. A Critical Review of Milk Fouling in Heat Exchangers. Comprehensive Reviews in Food Science and Food Safety. Institute of Food Technologists. (5): p. 27 -33, 2006.

BREMER, P.J., FILLERY, S. \& McQUILLAN, A. J. Laboratory scale Clean-In-Place (CIP) studies on the effectiveness of different caustic and acid wash steps on the removal of dairy biofilms. International Journal of Food Microbiology, v.106 , p. 254 - 262, 2006.

CHEN, X. D., ÖZKAN, N., QIN, F., XIN, H. \& LIN, L. An Effective CIP Procedure for Removing Dairy Protein Based Deposit - A Laboratory Investigation. 2003 ECI Conference on Heat Exchanger Fouling and Cleaning: Fundamentals and Applications. New Mexico, USA.

GARCIA, C. Modelagem e Simulação de Processos Industriais e de Sistemas Dinâmicos. 2005. EDUSP, São Paulo.

GEORGIADIS, M. C., ROTSTEIN, G. E. \& MACCHIETTO, S. 1998. Modeling and Simulation of Shell and Tube Heat Exchangers under Milk Fouling. AIChe Journal. Vol. 44, No. 4: 959 - 971.

GORMEZANO, L. 2007. Desenvolvimento e implementação de sistema para avaliar a cinética de remoção de resíduos presentes nos tubos de trocador de calor feixe tubular. 
Dissertação (Mestrado). São Caetano do Sul. Centro Universitário do Instituto Mauá de Tecnologia.

OGATA, K. Engenharia de Controle Moderno. Rio de Janeiro: Prentice Hall do Brasil, $3^{\text {a }}$ edição, 2000.

REIS, A. P. P., NEIRO, S. M. da S., COUTINHO FILHO, U., KUNIGK, L. E GEDRAITE, R. " Contribuição ao estudo da cinética da remoção de resíduos na etapa de enxágue do processo de limpeza CIP visando a minimização de gastos com insumos: Ajuste de modelos aos dados experimentais relacionados à variação de $\mathrm{pH}$ com a vazão". In: Anais do XVII Jornada em Engenharia Química,Uberlândia/MG, 2012.

SUNDARESAN, K.R. e KRISHNASWAMY, P.R. Estimation of time delay, time constanst parameters in time, frequency and Laplace domains. The Canadian Journal of Chemical Engineering. 1978, Vol. 56, 2, pp. 257-62 